\title{
Chromosome Survey of Some Australian Tettigoniids (Orthoptera-Tettigonioidea): Two Species with Neo-XY Sex-Determining Mechanism
}

\author{
Amilton Ferreira ${ }^{1}$ \\ Departamento de Biologia Geral da Faculdade de Filosofia, Ciencias \\ e Letras de Rio Claro, São Paulo, Brasil
}

Received August 30, 1968

\section{Introduction}

The superfamily Tettigonioidea comprises more than 3,500 species (Asana et al. 1938) of which nearly 100 species have been hitherto studied cytologically. Very little progress has so far been made in the chromosome survey of these insects as compared to other orthopteran insects (Asana et al. 1938). Up to date, the situation has not changed and few species have been studied in the last ten years. This group of insects is characterized by a variation in its chromosome number that extends over a wide range. Most of the species have males with an $\mathrm{XO}$ sex-chromosome mechanism, the $\mathrm{X}$ being either acrocentric or metacentric and always heteropycnotic during first prophase, its size being rather variable among the species (McClung 1902, 1908, Winiwarter 1931, Asana et al. 1938, White 1941, Piza 1950, 1958, Bianchi 1966).

Only four species are at present known to have changed their XO mechanisms to a more complicated system. McClung $(1902,1905,1914)$ was probably the first cytologist who found an XY 1 -XX우 species in tettigoniids. Due to poor knowledge of the sex chromosome mechanisms at that date, we cannot obtain helpful information about the species studied by him. However, it was assumed (White et al. 1967) from his drawings that Anabrus simplex is a species with $\mathrm{XY}$ males, although a reinvestigation of the karyotype of this species is desirable. 63 years after McClung's publication, Dave (1965) described a species with XY males (Isopsera sp.) and another with $\mathrm{X}_{1} \mathrm{X}_{2} \mathrm{Y}$ males (Letana atomifera), both from India. More recently, White et al. (1967) have described two more species with XY males, one of them from Australia and the other from South America.

In an attempt to contribute to a better understanding of the evolution of the long-horned grasshoppers and especially of the Australian species which are cytologically almost unknown, we started to investigate the karyotype of

1 This work was carried out at the Department of Genetics at the University of Melbourne, where the author was a Post-Doctoral Fellow, sponsored by "Campanha Aperfeiçoamento Pessoal Escola Superior" (C.A.P.E.S.) and "Fundação Amparo Pesquisa Estado São Paulo" (F.A.P.E.S.P.). 
some species that we have collected in Victoria and New South Wales. In this paper we deal with eleven species, two of them with XY males.

\section{Material and methods}

The specimens studied in this paper were collected by the author at the following localities :

\section{Phaneropterinae}

Polichne parvicauda (Stål)

No. 6, Sugarloaf Flat, below Falls 300, Gibraltar Creek, New South Wales

No. 24, 13 miles North Wheeny Creek, New South Wales

Nos. 18, 19, Warburton, Victoria

Tinzeda albosignata (Brunn.)

Nos. 25, 26, 10 miles south of Geranium, South Australia

Torbia viridissima (Brunn.)

Nos. 15, 16, Warburton, Victoria

Caedicia marginata (Brunn.)

Nos. 7, 8, Wheeny Creek, Blue Mountains, New South Wales

Caedicia sp. (a)

Nos. 9, 10, 11, vicinity of Milbrodale, New South Wales

Caedicia sp. (b)

No. 14, vicinity of Warburton, Victoria

Caedicia sp. (c)

No. 20, vicinity of Warburton, Victoria

Listroscelinae

Yorkiella sp. 2.

Minnie Creek, near Virginia Range, Western Australia (collected by M. J. D. White)

Conocephalinae

Anelitra sp.

Nos. 28, 29, Sherbrooke Forest, Dandenong Range, Victoria

Genus .A. sp. A.

Nos. 30, 31, Sherbrooke Forest, Dandenong Range, Victoria

Lanciana albidicornis

\section{Decticinae}

No. 5, 13 miles North Wheeny Creek, New South Wales

All the testes were fixed in a mixture of ethyl alcohol-acetic acid (3-1). The squash preparations of the chromosomes were stained by the Feulgen method. (Only testes of Anelitra sp. and Genus A sp. A were sectioned in order to study the real position of the chromosomes on the metaphase plate).

The specimens used in this work will be deposited in the Australian National Insect Collection in Canberra, Australia, with the numbers mentioned above.

\section{Observations}

Polichne parvicauda (Stål)

The specimens identified as Polichne parvicauda, have shown two dif- 
ferent karyotypes and due this fact they will be described separately. They will be distinguished by the number given to the specimens deposited in the A. N. I.C. in Canberra.

Specimen No. 6.

The diploid number of chromosomes as seen at spermatogonial metaphase is 16. There are five metacentric elements, four of which are autosomes, one being the $\mathrm{X}$ chromosome. The remaining eleven chromosomes are acrocentric, including the $\mathrm{Y}$.

In the primary spermatocytes, seven pairs of autosomal bivalents are formed (Fig. 1 and 2). ${ }^{2}$ They can be divided according to length into three groups: one large pair (1), a
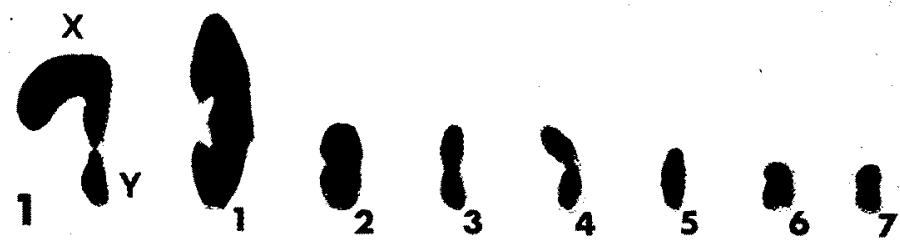

Fig. 1. Specimen 6 of Polichne parvicauda. 1, first metaphase chromosomes arranged in order of size. Sex chromosomes at the group of three left-hand end.

medium sized pairs $(2,3,4)$, and three small pairs $(5,6,7)$. The pairs numbered 1 and 2 are metacentric autosomes, while the remaining five pairs are formed by acrocentric elements. Two distal chiasmata, one in each arm, occur between the metacentric chromosomes, while only one occurs between the acrocentric ones. The pair numbered 1 has unequal arm lengths, the longer arm being twice the length of the shorter. The pair numbered 2 is metacentric with approximately equal arm lengths.

The sex-determining mechanism is clearly of the neo-XY $\delta$ type. The neo-X is metacentric and the biggest element of the karyotype. ts arms are of different sizes, $\mathrm{XL}$ being at least twice the length of XR. The neo-Y is acrocentric, medium-sized and at first metaphase shows approximately the same length as XR (Fig. 3).

In the prophase of the first meiotic division, we can clearly see that there is pairing between XR and Y (Fig. 4). XL is the only element with heteropycnosis in the first prophase of the first meiotic division. During first metaphase, the $\mathrm{X}$ and $\mathrm{Y}$ are linked by an interstitial chiasma (Figs. 6 and 7) and remain together until early anaphase at which time they segregate and move to opposite poles giving rise to two kinds of secondary spermatocytes (Fig. 5). Both have the same chromosomal number but differ from each other by the presence of either the $\mathrm{X}$ or the $\mathrm{Y}$ (Fig. 5).

1 All the photographs with the exception of Fig. 18 were exposed at a magnification $\times 1,800$. Fig. 18 was exposed at a magnification of $\times 795$. 


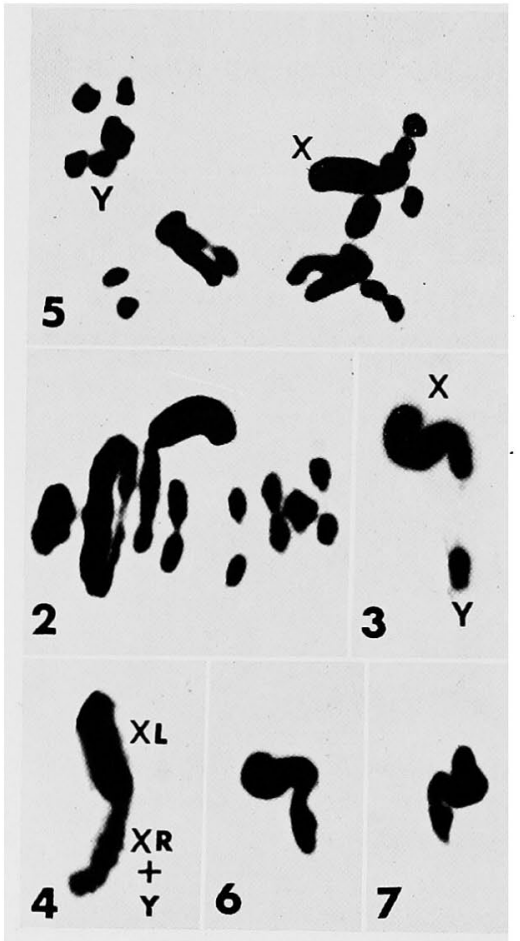

Figs. 2-5. 2, first metaphase. 3, the sex bivalent during early anaphase. 4, the sex bivalent during pachytene. 5, late anaphase showing the two kinds of secondary spermatocytes formed. $6-7$, sex bivalent in first metaphase showing an interstitial chiasma.

Specimens no. 18, 19, and 24.

These individuals have $2 n=21 \hat{\delta}$. All the chromosomes are acrocentric and the sex-determination is of the XO type. At spermatogonial metaphase, eighteen small autosomes plus two large ones, as well as the $\mathrm{X}$, are seen. The $\mathrm{X}$ is slightly larger than the largest autosome pair (Fig. 8).

During the first metaphase, the nine pairs of small bivalents show one chiasma each, while the biggest bivalent shows two chiasmata (Fig. 9). During the anaphase, it was observed that the biggest pair has a delayed segregation when compared with the remaining bivalents (Fig. 10).

\section{Caedicia marginata Brunn.}

This species has a neo-XY sexchromosome mechanism like Polichne parvicauda, but there are some differences between them. In C. marginata, the diploid number of chromosomes is $20 \hat{\delta}$ as seen at spermatogonial metaphase (Fig. 11). With the exception of the neo-X, which is metacentric, the remaining chromosomes are acrocentric. In the primary spermatocytes nine pairs of autosomal bivalents are formed; they

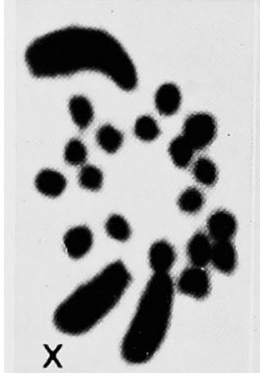

8

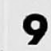

9
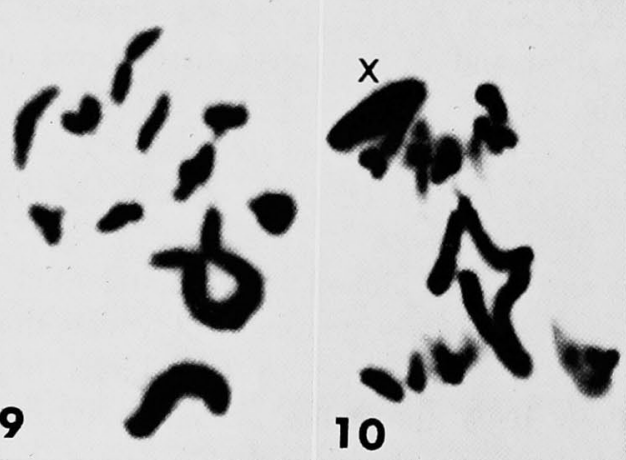

Figs. 8-10. Specimen 18 of Polichne parvicauda. 8, spermatogonial metaphase. 9, diakinesis. 10, late anaphase. can be grouped into three pairs of large chromosomes, five pairs of medium-sized and one pair of small elements (Figs. 12 and 13). Only one chiasma is observed in each pair, except the largest bivalent which shows two chiasmata.

The $\mathrm{X}$ chromosome is the only metacentric element of the set and it is 
also the largest one. $\mathrm{XL}$ and XR are of different lengths, the first being twice as big as the second, and the only heteropycnotic element during the prophase of the first meiotic division. The $\mathrm{Y}$ being acrocentric and medium-sized is very difficult to recognize at spermatogonial metaphase. During early anaphase we can clearly see that $\mathrm{XR}$ and $\mathrm{Y}$ have the same length.

The chiasma

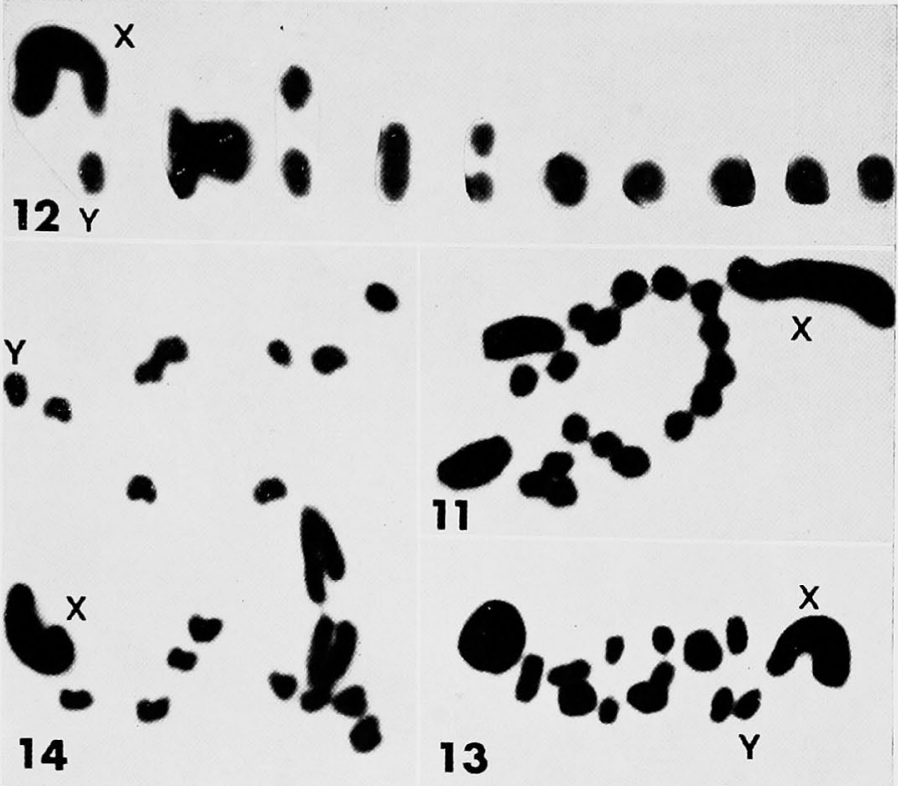

Figs. 11-14. Caedicia marginata. 11, spermatogonial metaphase. 12, first metaphase chromosomes arranged in order of size. Sex chromosome at the left-hand side. 13, first metaphase. 14, late first anaphase showing the two kinds of secondary spermatocytes formed. that links the neo$\mathrm{X}$ and the neo-Y persists until early anaphase when these chromosomes, like those of the specimen number 6 of $P$. parvicauda, segregate and move to different poles together with two or three pairs of autosomes ahead of the remaining pairs. Two kinds of secondary spermatocytes, like those in the above mentioned species are formed (Fig. 14).

Caedicia sp. a, Caedicia sp. b, Caedicia sp. c.

These species have $2 n=21 \hat{\delta}$. All the chromosomes are acrocentric and the sex determination is of the XO type. The size of the chromosomes, the number of chiasmata as well as the delayed segregation of the biggest pair was found to be similar to those observed in specimens nos. 24, 18 and 19 of Polichne parvicauda.

\section{Tinzeda albosignata (Brunn.)}

The chromosome number as seen at spermatogonial metaphase is $25 \hat{0}$, all the elements being acrocentric (Fig. 15). The sex mechanism is of the $\mathrm{XO}$ type, with the $\mathrm{X}$ chromosome being slightly smaller than the biggest autosome.

During first metaphase, twelve bivalents are formed, besides the unpaired $\mathrm{X}$. Eight bivalents are small and three are medium-sized, all of them with 


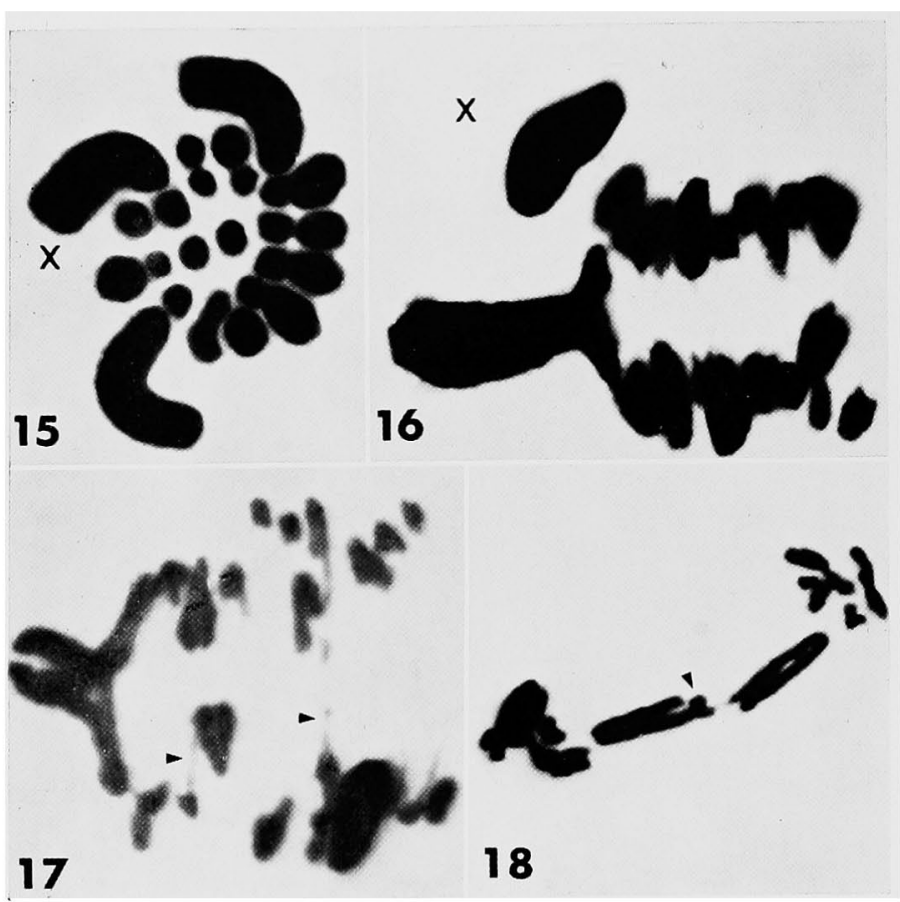

Fig. 15-18. Tinzeda albosignata. 15, spermatogonial metaphase. 16 , late anaphase showing the biggest pair still paired. 17, late anaphase showing some bridges (arrowed). 18, late anaphase showing a breakage in one chromatid (arrowed).

small variation in length. The re maining bivalent and the $\mathrm{X}$ are no. ticeable among the other due to their enormous size, the desynchronized mechanical behav. iour and also a high number of chiasmata on the biggest pair (Fig. 16). The $X$ always migrates towards a pole ahead of the remaining chromo somes and between the autosomes, the biggest pair is al. ways delayed in its segregation. starts to segregate when the $\mathrm{X}$ and the other chromosomes have already reached the poles.

A few anaphases showed bridges between the separating chromosomes in the case of the small bivalents and the largest one (Fig. 17). In the cast of one bridge between the longest chromosomes, a break in one chromatic was observed (Fig. 18).

\section{Genus A. sp. a}

The spermatogonial complement of this species is $2 n=29 \hat{\circ}$ with an XC sex-determining mechanism.

At the spermatogonial metaphase, we can see that there is a sharr bimodality in lengths among the autosomes, with the largest pair bein twelve times larger than the smallest (Fig. 19). They can be classified a follows : 7 pairs of small and acrocentric elements; 6 pairs of medium-sized which are also acrocentric; and 1 pair of large metacentric chromosomes The $\mathrm{X}$ is acrocentric and larger than each of the acrocentric autosomes, but it is smaller than the metacentric pair.

At the first metaphase (Fig. 20), 14 bivalents are formed, among whicl one is prominent due to its relatively enormous size and high frequency o chiasma. In squashed cells, it appears to lie in a different plane than do th other chromosomes. However, examination of sections did not show an: special location in relation with the equatorial plate. 
During the first anaphase, we can see that there is a desynchronysed movement of the chromosomes to the poles (Fig. 21). When the X chromosome and the small and medium-sized autosomes had already segregated and reached the poles, the largest pair was still linked by a terminal chiasma in the middle of the cell.

\section{Anelitra sp.}

The chromosome number of this species is similar to that described for Genus A. sp. a with $2 n$ =29合 (Fig. 22). The main difference was found

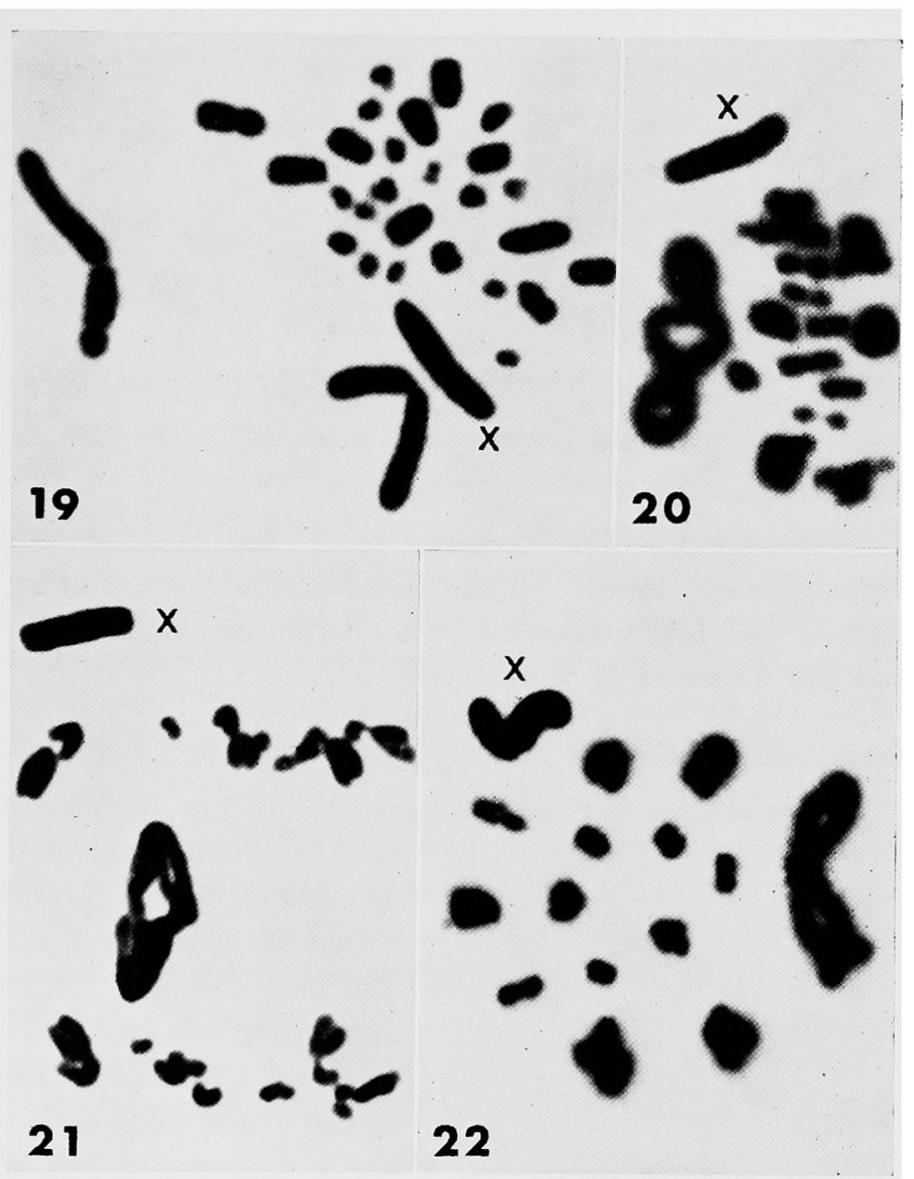

Figs. 19-22. Genus $A s p . a$. 19, spermatogonial metaphase. 20, first anaphase showing the biggest bivalent with four chiasmata. 21 , late first anaphase with a delayed segregation of the biggest pair. 22. Anelitra sp. diakinesis.

in the $\mathrm{X}$ chromosome since in Anelitra sp. this chromosome is metacentric and larger than in Genus A. sp. a.

The difference in lengths among the autosomes, the high number of chiasma found in the largest metacentric pair and also the desynchronized movement during first anaphase were similar to those observed in Genus A. sp. a.

\section{Torbia viridissima (Brunn.)}

The two specimens studied have $2 n=19 \hat{\delta}$ acrocentric chromosomes, with an XO sex mechanism (Figs. 23-23A). At spermatogonial metaphase, fourteen small autosomes, two medium-sized and two big chromosomes, as well as the X, are seen. Unfortunately, first metaphases were not observed but diakineses with eight small bivalents and a medium-sized one having one chiasma each and one big bivalent with two chiasmata, were observed. The 


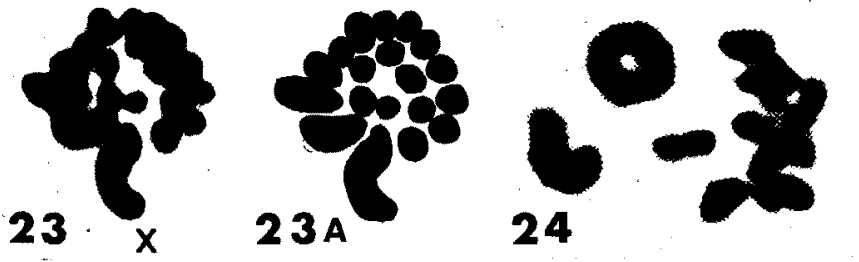

Figs. 23-24. Torbia viridissima. 23, spermatogonial metaphase. 24, diakinesis.
$\mathrm{X}$ chromosome is unpaired (Fig. 24). As in the species described above, the biggest pair also has a delayed segregation at first anaphase.

Yorkiella sp. 2

This species, like Yorkiella sp. 1, has $31 \hat{\delta}$ chromosomes with an XO sex-mechanism type. All the autosomes are acrocentric, while the $\mathrm{X}$ is metacentric. At first metaphase seven large bivalents, eight medium-sized ones and the unpaired $\mathrm{X}$ were seen.

\section{Lanciana albidicornis}

This species has $2 n=25 \hat{\delta}$, with all the chromosomes acrocentric and an XO sex mechanism (Fig. 25). At the first

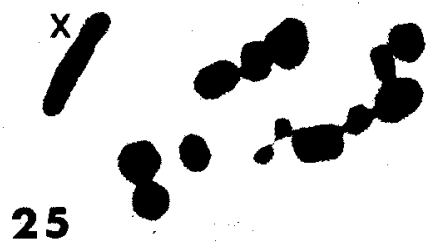

Fig. 25. Lanciana albidicornis. First metaphase. metaphase, twelve bivalents besides the unpaired $\mathrm{X}$ were seen. They can be divided into one group of six small bivalents, and one group of six medium-sized ones. The main characteristic of this species is that there is no sharp difference in the chromosome size within each group or even between both groups.

\section{Discussion}

The origin of the neo-XY sex-chromosome mechanism in the specimen number 6 of Polichne parvicauda and in Caedicia marginata does not differ in principle from those already described by Dave (1965) and by White et al. (1967) in other species. There was a centric fusion between a long acrocentric $\mathrm{X}$, which is totally heteropycnotic, with one of the small autosomes. The unfused autosome has become a neo-Y.

In spite of the fact that we did not find a great number of cells in first prophase stage, some conclusions can be made about the sex mechanisms of both species, especially in the specimen numbered 6 of Polichne parvicauda. They seem to have acquired this mechanism recently and this is more obvious in $P$. parvicauda than in C. marginata. This statement is supported by the following observations :

a) $\mathrm{XR}$ and $\mathrm{Y}$, during prophase of the first meiotic division, are paired throughout their total length and do not develop any sign of hetero- 
chromatinization ;

b) during early anaphase $\mathrm{XL}$ and $\mathrm{Y}$ are equal in length;

c) interstitial chiasmata were observed between the former autosomes.

The information about Caedicia marginata is poorer and we can only say that $\mathrm{XR}$ and $\mathrm{Y}$ are of the same size, and that they do not show any heterochromatinization during first prophase and their segregation is ahead of the autosomes.

Polichne parvicauda does not seem to have undergone any later change in the former pair of autosomes that was incorporated into the sex system. This fact leads us to place the sex mechanism of this species in an early stage of evolution, close to that described in Yorkiella picta. Theudoria melanocnemis (White et al. 1967) and Isopsera sp. exhibit a much later stage in the evolution of the neo-XY mechanism. Some of the changes they have undergone, like the difference in length between $\mathrm{XR}$ and $\mathrm{Y}$ and even the heterochromatinization of the $\mathrm{Y}$, were not observed in $C$. marginata This prompted us to include this species in a group that is closer to $Y$. picta and P. parvicuuda.

The differences observed in the karyotypes of the specimens classified as Polichne parvicauda cannot at the moment be understood; we are not able to say whether it is a polymorphic species or group of sibling species. However, considering that the Australian tettigoniids have received very little study, we must consider the possibility that we have two different species. Cytological and taxonomic studies of a greater number of specimens must be carried out to clarify the situation.

The chromosome number within the subfamily Phaneropterinae varies from 16 to 33 and some species have been reported as having 25, 2729 and 31 chromosomes. At the moment, it is not possible to say which is the basic karyotype for the subfamily and more cytological work associated with systematics should be done. However, some variation found by us in Polichne parvicauda and in Torbia viridissima can be easily explained as having arisen from karyotypes that are basic for some groups of species. Species with $2 n \hat{\delta}=21$ have all their chromosomes acrocentric, the $\mathrm{X}$ being the biggest element of the set. Besides the $\mathrm{X}$, only one pair of autosomes can be easily distinguished by its size, while the others are uniform in their length. These facts are easily seen in the Australian species of the genus Caedicia we have been studying.

Torbia viridissima has undergone a reduction of its chromosome number from $2 n=21$ to $2 n=19$ and both species have an acrocentric medium-sized chromosome that was not observed in the species having $2 n=21$. We suspect that an integration of chromatin took place to form this chromosome and, as a consequence, the reduction in number occurred. However, the biggest reduction was found in specimen number 6 of Polichne parvicauda which has $2 n=16$. We have already mentioned that in this species there was a 
centric fusion between the former $X$ and a small autosome giving rise to a neo-XY sex-chromosome mechanism. But in this case, some centric fusions between autosomes have also taken place. One of them was between the largest pair and one of the small ones, giving rise to a submetacentric chromosome. The other was between two of the small chromosomes, producing a medium-sized metacentric element.

The autosomal fusion that we have found in $T$. viridissima and $P$. parvicauda cannot be associated as they have different origins. The $\mathrm{X}$ autosomal fusions in $C$. marginata and $P$. parvicauda cannot be related as they were found in species taxonomically separated, even though they have a similar mode of origin.

The asynchronized mechanical behaviour of the autosomes that we have found in all species so far studied, except Lanciana albidicornis, is related to a sharp difference in size between them. A high chiasma frequency was found in the largest bivalent. This leads to a late terminalization of Eits chiasmata and as a consequence to an asynchronized anaphase.

Bimodality of chromosome size associated with errors confined to the long chromosome was described by John et al. (1960) on Chorthippus brunneus.

Species such as L. albidicornis where the chromosomes do not show a great difference in size, achieve a synchronized mechanical behaviour more easily.

The first few anaphases found in $T$. albosignata do not allow us to determine the nature or the frequency of the bridges observed in three of its small bivalents and in the largest one. However, there seems to be a localized association of sister chromatids like those found by Henderson (1961) in Pholidoptera griseoaptera and by John et al. (1960) in Chorthippus brunneus.

No different degree of coiling was observed by us in the bivalents involved as was found Pholidoptera and one bridge with a distal breakage was observed. Nevertheless, we are not able to say whether the fragment was still attached to its chromatids.

\section{Summary}

The chromosome number of 11 species of Australian tettigoniids, (Orthoptera-Tettigonioidea) are reported in this paper. The main characteristics of the karyotype of these species are given in the following table.

In Polichne parvicauda (specimen no. 6) and Caedicia marginata a centric fusions between the former $\mathrm{X}$ and a small autosome was found. These fusions gave rise to a neo-XY sex-determining mechanism in both species. Besides, two centric fusions between autosomes occurred in the former species, forming two pairs of metacentric chromosomes and reducing the chromosome number to $2 n=16$.

In Torbia viridissima a reduction to $2 n=19$ due to an integration of 


\begin{tabular}{l|c|c}
\hline \multicolumn{1}{c|}{ Species } & Chromosome number 2n-ठ & Sex mechanism \\
\hline Polichne parvicauda 24, 18, 19 & 21 & $\mathrm{XO}$ \\
Polichne parvicauda 6 & 16 & $\mathrm{XY}$ \\
Caedicia sp. a. & 21 & $\mathrm{XO}$ \\
Caedicia sp. b. & 21 & $\mathrm{XO}$ \\
Caedicia sp. c. & 21 & $\mathrm{XO}$ \\
Caedicia marginata & 20 & $\mathrm{XY}$ \\
Genus A. sp. a. & 29 & $\mathrm{XO}$ \\
Anelitra sp. & 29 & $\mathrm{XO}$ \\
Tinzeda albosignata & 25 & $\mathrm{XO}$ \\
Torbia viridissima & 19 & $\mathrm{XO}$ \\
Yorkiella sp. 2 & 31 & $\mathrm{XO}$ \\
Lanciana albidicornis & 25 & $\mathrm{XO}$ \\
\hline
\end{tabular}

chromatin between apparently two small autosomes took place.

The desynchronized movement of the chromosomes during first anaphase due to their bimodality in length as well as the difference in chiasma frequency was discussed.

\section{Acknowledgments}

The author is greatly indebted to Professor M. J.D. White and Ing. Agr. Alejo Mesa (Melbourne University) for the critical reading of the manuscript and to Dr. K.H.L. Key (Division of Entomology, C.S.I.R.O., Canberra) for the identification of the specimens.

\section{Literature cited}

Asana, J. J., Makino, S. and Niiyama, H. 1938. A chromosomal survey of some Indian insects I. Morphology of the chromosomes in eight species of the Locustidae. J. Fac. Sci. Hokkaido Univ. Ser. VI 6: 221-234.

Bianchi, P.A. 1966. Note sulla cariologia di alcuni Efippigeridae (Insecta-Orthoptera). Rend. Accad. Lincei. Fis. mat. anat. 41: 553-557.

Dave, M. J. 1965. On unusual sex chromosomes found in two species of the Locustidae. Cytologia 30: 194-200.

Henderson, S. A. 1961. Chromosome number and behaviour in the grasshopper Pholidoptera. Heridity 16: 181-186.

John, B., Lewis, K. R. and Henderson, S. A. 1960. Chromosome abnormalities in a wild population of Chorthippus brunneus. Chromosoma 10: 589-618.

Makino, S. 1950. An atlas of the chromosome number in animals. Iowa State College Press.

McClung, C.E. 1902. The spermatocyte divisions of the Locustidae. Kansas Univ. Sci. Bull. 1: 185-231.

- 1905. The chromosomes complex of orthopteran spermatocytes. Biol. Bull. Woods Hole 9: $301-340$.

- 1908. The spermatogenesis of Xiphidium fasciatum. Kans. Univ. Sci. Bull. 4: 253-262.

- 1914. A comparative study of the chromosomes in orthopteran spermatogenesis. J. Morph. 25: 651-749. 
Piza, S. de Toledo 1950. Breve noticia acerca dos cromossomios de Ischyra punctinervis Brunner e Phylophyllia guttulata Stảl. (Orthoptera, Phaneropterinae). Folia Clinica et Biologica 16(2): 93-95.

- 1958. A short note on the chromosomes of Phlugis (Orthoptera-Listroscelinae). Rev. Agric. (Piracicaba-Brazil). 330(2): 72-73.

White, M. J.D. 1941. The evolution of the sex chromosome II. The X-chromosome in the Tettigoniidae and Acrididae and the principle of evolutionary isolation of the $\mathrm{X}$. J. Genet. 42: 173-190.

-, Mesa, A. and Mesa, R. 1967. Neo-XY sex chromosome mechanism in two species of Tettigonioidea (Orthoptera). Cytologia 32: 190-199.

Winiwarter, H. de 1931. Evolution de l'heterochromosome chez Tettigonia (Decticus) albifrons (Fab.) Arch. Biol., Paris, 42: 201-228. 